\title{
Discrete Lattice Material Vacuum Airship
}

\author{
Benjamin E. Jenett* \\ Massachusetts Institute of Technology, Cambridge, MA, 02139 \\ Christine E. Gregg ${ }^{\dagger}$ \\ NASA Ames Research Center, Moffett Field, CA 95035 \\ Kenneth C. Cheung \\ NASA Ames Research Center, Moffett Field, CA 95035
}

\begin{abstract}
Vacuum airships fueled by renewable energy would reduce reliance on fossil fuel-based modes of transport, lessen the need for limited and non-renewable lifting gases, and can be achieved using novel manufacturing techniques for ultra-light, discrete lattice material systems. The Discrete Lattice Material Vacuum Airships (DLMVA) system combines novel material science and manufacturing technologies for new modes of mass transportation, resulting in a disruptive approach to reduce national resource consumption and emissions. Through the use of high performance building block elements, modular, scalable and extensible aircraft can be rapidly assembled into positive net-buoyancy systems utilizing a vacuum instead of a lifting gas. By using architected lattice material principles, show that lattice materials can overcome stability limitations of previous vacuum balloon designs. Additionally, we show that lattice vacuum balloons are strength limited, rather than stability limited. As a result, airborne infrastructure can be developed to support the proliferation of modern systems such as e-commerce and distributed communications, while simultaneously reducing dependence on finite, non-renewable, emission-heavy resources.
\end{abstract}

\section{Nomenclature}

\begin{tabular}{lll}
$A$ & $=$ lattice modulus linear factor \\
$B$ & $=$ lattice strength linear factor \\
$\bar{E}$ & $=$ lattice relative modulus \\
$E_{l}$ & $=$ lattice modulus \\
$E_{S}$ & $=$ lattice constituent solid modulus \\
$P_{\text {atm }}$ & $=$ atmospheric pressure \\
$P$ & $=$ pressure \\
$\delta P$ & $=$ infinitesimal pressure increase \\
$V$ & $=$ lattice volume under no pressure \\
$V_{l, 1 \text { atm }}$ & $=$ lattice volume at 1 atm hydrostatic pressure \\
$\delta V_{l, 1 \text { atm }}$ & $=$ infinitesimal volume increase \\
$\alpha$ & $=$ lattice modulus scaling factor \\
$\beta$ & $=$ lattice strength scaling factor \\
$\kappa$ & $=$ bulk modulus \\
$\bar{\rho}$ & $=$ relative modulus \\
$\rho_{\text {air }}$ & $=$ density of air \\
$\rho_{l}$ & $=$ lattice density \\
$\rho_{l, 1 \text { atm }}$ & $=$ lattice density at 1 atm hydrostatic pressure \\
$\rho_{s}$ & $=$ lattice constituent solid density \\
$\bar{\sigma}$ & $=$ relative strength \\
$\sigma_{l}$ & $=$ lattice strength \\
\hline${ }^{*}$ Graduate Researcher, MIT Center for Bits and Atoms, Cambridge, MA 02139 & \\
${ }^{\dagger}$ Research Scientist, NASA Ames Research Center Coded Structures Laboratory, Moffett Field, CA 95035 \\
${ }^{*}$ Research Scientist, NASA Ames Research Center Coded Structures Laboratory, Moffett Field, CA 95035
\end{tabular}


$\begin{aligned} \sigma_{S} & =\text { lattice constituent solid strength }\end{aligned}$

$\mu \quad=$ Poisson ratio

\section{Introduction}

Transportation accounts for nearly $30 \%$ of United States energy usage[1], with corresponding fuel costs and greenhouse gas $(\mathrm{GHG})$ emissions. Organizations relying on transportation are naturally compelled to find more efficient solutions. To this end, e-commerce companies have described loitering helium-filled airships as unmanned airborne warehouses and hubs for drone-delivery systems.[2] These types of e-commerce companies ship on the order of $10^{9}$ packages per year $\left(10^{6}\right.$ per day), with a significant reliance on truck transport. Transportation as a whole accounts for $27 \%$ of U.S. greenhouse gas (GHG) emissions (roughly 1.85 billion metric tons of $\mathrm{CO}_{2}$ equivalent) [3]. Of this, freight trucks account for 19\%, or 350 million metric tons. An integrated airship and rotorcraft approach could remove the "last mile" delivery reliance on traditional logistics companies who deliver via truck from a distribution center to the final destination. It is clear that if consumer package delivery transitions to more efficient transportation modes, a significant impact on cost, total energy consumption, and GHG production will be realized.

However, national fleets of helium airships pose a problem. For all its advantages as a light-than-air inert gas, helium has stalled the advancement of the airship industry. No materials exist that can prevent the diffusion of helium through an airship's envelope. This loss of helium leads to two effects: first, it leads to a loss of lift and hence a reduction in operational capability of the airship; second, it adds to the operational costs because the helium must be replaced to maintain operational capability. Although the U.S. has 34\% of the world's helium reserves [4], it is still a limited and non-renewable resource that cannot be practically manufactured from other materials; as it diffuses into the atmosphere, helium molecules escape into space and are lost.

If airborne infrastructure can lead to reduction of GHG emissions, then a scalable, sustainable system for airships must be developed. The development of a vacuum airship would free the U.S. airship industry from its dependence on helium and greatly reduce operational costs of airships. The vacuum airship would push the boundaries of sustained flight and drastically change the way the U.S. utilizes airships and their impact around the world.

The idea of a vacuum airship originated hundreds of years ago, but material limitations have so far rendered them infeasible. However, recent advances in materials science, particularly in the field of architected materials, prompt a return to the concept. A patent was filed in 2006 for a sandwich structure, thin-shelled, spherical vacuum balloon, constructed from a cellular solid core [5], but manufacturing remains a significant challenge. In this work, we re-evaluate theoretical feasibility of various vacuum balloon designs in light of recent advances in advanced architected cellular materials. We then present an initial design based on discrete lattice materials.

\section{Method}

\section{A. Cellular Materials Background}

The invention of artificial foams decreased by orders of magnitude the lowest density material available to designers [6]. These and other cellular solids such as honeycombs are already used in mass-critical structural applications in the form of core for sandwich structures. The mechanical properties of both random and periodic cellular solids can be predicted based on the mechanical properties of the constituent material and the relative density, or volume fraction, of the cellular solid [7]. For example, the modulus of of a cellular solid can be predicted according to the relation:

$$
\bar{E}=\frac{E_{l}}{E_{s}}=A \bar{\rho}^{\alpha}
$$

where $\bar{E}$ is relative modulus, the modulus of the lattice $E_{l}$ normalized by the modulus of the constituent solid $E_{s}$. $A$ and $\alpha$ are constant scaling factors dependent on cellular geometry. The relative density, $\bar{\rho}$, is the density of the lattice $\rho_{l}$ normalized by the density of the constituent solid $\rho_{s}$, and can be written as

$$
\bar{\rho}=\frac{\rho_{l}}{\rho_{s}}
$$

A similar scaling relationship was established for relative strength, the strength of the lattice $\sigma_{l}$ normalized by constituent material strength $\sigma_{s}$,

$$
\bar{\sigma}=\frac{\sigma_{l}}{\sigma_{s}}=B \bar{\rho}^{\beta}
$$


The scaling factors $B$ and $\beta$ are dependent upon both cellular geometry and relative density regime. Random foams and other bend dominated geometries [8] are known to have scaling factors $\alpha$ and $\beta$ greater than or equal to 2, corresponding to two orders of magnitude reduction in relative performance for every single order of magnitude reduction in relative density. By controlling the microstructure of the cellular solid and choosing stretch dominated geometries, architected cellular materials exceed the performance of random or bend dominated geometries and achieve linear scaling ( $\alpha$ and $\beta$ equal to 1) [9]. Accordingly, architected materials have demonstrated previously unprecedented stiffness and strength at very low densities [9.-11]. Additionally, advances in the field of discrete lattice materials have shown improved manufacturability for these materials [10, 11], providing a pathway towards adoption into large-scale applications. Importantly, discrete lattice materials have shown scalable manufacturing of architected lattices using high-performance, uni-directional carbon fiber [12].

Vacuum balloons require high strength and stiffness at extremely low densities, making them an appealing application for architected lattice materials. To assess the influence of architected materials on vacuum balloon feasibility, we first evaluate theoretical performance of various vacuum balloon designs based on an ideally performing isotropic lattice [13]. Informed by the theoretical performance of an ideal sphere from an ideal lattice, we seek to perform an initial design study with discrete lattice materials to assess feasibility. The focus on discrete lattice materials responds to traditional manufacturing challenges, and limits designs to those that can be feasibly constructed from a building-block approach [11, 12]. The focus of this design study is to evaluate whether viable designs can be achieved given the geometry constraints of discrete lattice materials and validate theoretical predictions using simulation.

\section{Analysis}

The following analyses evaluate the theoretical feasibility of a solid, thick-shell, and thin-shell lattice vacuum airship. For the purposes of theoretical analysis, several assumptions are made for all designs. Each design is assumed to be a spherical vessel under sea level standard atmospheric conditions, atmospheric pressure $P_{\text {atm }}$ of $101 \mathrm{KPa}$ and air density $\rho_{\text {air }}$ of $1.225 \mathrm{~kg} / \mathrm{m}^{3}$. As noted by [14], the critical buckling pressure of a spherical shell is highly sensitive to imperfections. For this analysis, we will use the ideal critical buckling pressure

$$
P_{c r i t}=\frac{2 E t^{2}}{\sqrt{3(1-v)} R^{2}}
$$

Any increase in this value associated with imperfections should be accounted for in safety factors during a detailed design phase.

We assume that we are able to fill each design with an ideal, isotropic lattice [13, 15]. This ideal lattice assumes linear power scaling of both modulus and strength with relative density ( $\alpha=1$ and $\beta=1$ ), as well as $A$ and $B$ equal to 0.1 in equations 1 and $2[9,11]$. At very low relative densities, it is common for the strength scaling coefficient $\beta$ of even stretch dominated lattices to shift from 1 to 2 . However, literature suggests that 0.001 is an approximate inflection point for the octet lattice [9], so we will continue to use the ideal parameter $\beta=1$ for relative densities considered above that order. For the purpose of this analysis, we assume a lattice Poisson ratio $\mu$ of 0.3 . Though this value is geometry dependent for various lattice materials, it can be shown that changes in this value have a weak influence on the results.

\section{A. Solid Sphere}

When designing vacuum balloons with cellular materials, a first natural question is whether there exists a cellular material that is light, stiff, and strong enough so that any solid block, once put under vacuum with a skin, would float. In other words, does there exist a cellular material such that the density

$$
\rho_{l, 1 \text { atm }} \leq \rho_{\text {air }}
$$

where $\rho_{l, 1 \text { atm }}$ is the density of the lattice at atmospheric of hydrostatic pressure and $\rho_{\text {air }}$ is the density of the air. $\rho_{l, 1 \mathrm{~atm}}$ can be written as $m / V_{l, 1}$ atm where $m$ is the mass of the lattice and $V_{l, 1 \mathrm{~atm}}$ is the volume of the evacuated lattice under atmospheric pressure. This can be related to the density of the lattice under no load using the bulk modulus $\kappa$, or the ratio of infinitesimal pressure increase to relative decrease in volume.

$$
\kappa=\rho \frac{d P}{d \rho}=\frac{\delta P}{\delta V / V}
$$




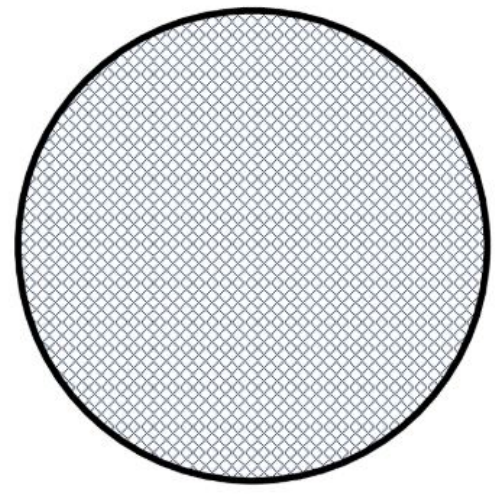

Solid

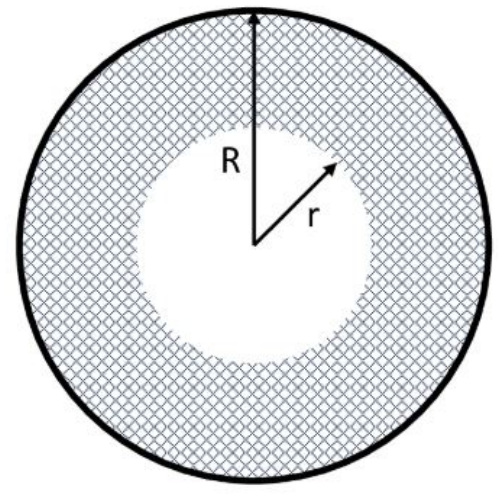

Thick Shell

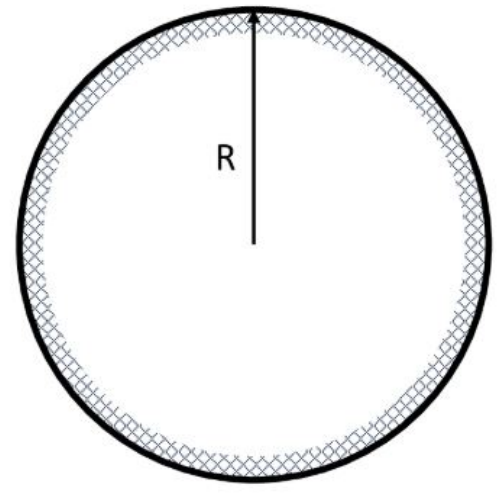

Thin Shell

Fig. 1 Schematic design of airships studied.

where $\mathrm{P}$ is pressure and $\mathrm{V}$ is the original volume at no pressure, or $V_{l, 1 a t m}=V-\delta V$. The density requirement can then be rewritten as

$$
\frac{m}{V-\frac{\delta P V}{\kappa}} \leq \rho_{\text {air }}
$$

Simplified and in terms of density, this can be written as

$$
\frac{\rho_{l}}{1-\frac{P_{\text {atm }}}{\kappa}} \leq \rho_{\text {air }}
$$

If we assume that a lattice material can be made such that it is approximated by a homogeneous, isotropic linear elastic solid,

$$
\kappa=\frac{E}{3(1-2 v)}
$$

where $E$ is the Young's modulus and $v$ is the Poisson ratio. Using this, the density relation can be rewritten

$$
\frac{E_{l} \rho_{l}}{E-3 P_{a t m}(1-2 v)} \leq \rho_{\text {air }}
$$

where $E_{l}$ is the modulus of the lattice. Using 1 to write $E_{l}$ in terms of the relative density, the relation can be rewritten for a cellular solid such that

$$
\frac{E_{s} \rho_{s} A \bar{\rho}^{2 \alpha}}{E_{s} A \bar{\rho}^{\alpha}-3 P_{\text {atm }}(1-2 v)} \leq \rho_{\text {air }}
$$

To evaluate the feasibility of this design with available materials, assume ideal lattice behavior and atmospheric pressure conditions. Evaluating 10 using these parameters,

$$
E_{s} \bar{\rho}\left(\rho_{\text {air }}-\rho_{s} \bar{\rho} \geq 1.484 M P a\right.
$$

For this to be true,

$$
\rho_{s} \bar{\rho}<\rho_{\text {air }}
$$

As a constituent material, it is advantageous to choose a material with a high stiffness to density ratio. Carbon fiber composites are widely available with densities less than $1100 \mathrm{~kg} / \mathrm{m}^{3}$. Therefore, a relative density of approximately 0.001 is required. Substituting this value into 11 , we arrive at a necessary constituent material stiffness of

$$
E_{s}>11.9 \mathrm{GPa}
$$

The modulus of typical carbon fiber composites is an order of magnitude higher than this requirement, thus fulfilling the stiffness and density requirements. 
Investigating the strength requirement, we find that the lattice must be strong enough to resist the pressure load,

$$
\sigma_{l}>P_{a t m}
$$

Substituting the expression for lattice relative strength from 2

$$
B \sigma_{s} \bar{\rho}^{\beta}>P_{\text {atm }}
$$

Evaluating the strength requirement for ideal lattice with the estimated necessary relative density of 0.001 , we find that

$$
\sigma_{s}>1.01 G P a
$$

We conclude that a solid cellular solid balloon is limited by the strength.

\section{B. Thick-Shell Balloon}

Next, we evaluate a thick-shelled spherical vessel, with outer radius $R$ and inner radius $r$. The fundamental mass requirement of a vacuum balloon, that the mass of the evacuated balloon weigh less than the volume of the displaced air, can be written as

$$
\rho_{l}\left(R^{3}-r^{3}\right) \leq \rho_{\text {air }} R^{3}
$$

Using the definition of relative density, this expression can be rewritten as

$$
\rho_{s} \bar{\rho}\left(R^{3}-r^{3}\right) \leq \rho_{\text {air }} R^{3}
$$

For now, we neglect the change in the volume of displaced air due to the compression of the sphere.

The shell must meet buckling stability and strength requirements. Thin-shell theory for the critical buckling pressure of a sphere is known to over-estimate that for a thick-shell, by $23.7 \%$ at $r / R=0.4$. However, we will use the thin shell theory as a conservative estimate, which we will later see suffices for this analysis. The thin-shell critical buckling pressure, $P_{\text {crit }}$ must be greater than the air pressure, $P_{\text {air }}$, such that

$$
P_{\text {crit }}=\frac{2 E_{l} t^{2}}{\sqrt{3(1-v)} R^{2}} \geq P_{\text {air }}
$$

where $E_{l}$ is the stiffness of the lattice and $t$ is the thickness of the shell. However, because we are dealing with a cellular solid, we can redefine the stiffness of the lattice as a function of the stiffness of the constituent material, $E_{S}$, and the lattice relative density, $\bar{\rho}$. Therefore, while also recognizing that $t=R-r$, we can rewrite 19 as

$$
\frac{2 E_{S} A \bar{\rho}^{a}(R-r)^{2}}{3\left(1-v^{2}\right) R^{2}} \geq P_{\text {air }}
$$

We derive our strength constraint from the maximum stress of a spherical thick-shelled pressure vessel, $\sigma_{\max }$. The maximum stress must be less than the strength of the cellular solid, $\sigma_{l}$, such that

$$
\sigma_{\text {max }}=\frac{3 P_{\text {air }} R^{3}}{2\left(R^{3}-r^{3}\right)} \leq \sigma_{l}
$$

However, just as with the stiffness, we can rewrite the strength of a cellular solid in terms of the strength of the constituent material, $\sigma_{s}$, and the lattice relative density. Therefore, we can rewrite 21 as

$$
\frac{2\left(R^{3}-r^{3}\right) \sigma_{s} B \bar{\rho}^{b}}{3 R^{3}} \geq P_{\text {air }}
$$

Equations 18, 20, 22 represent the most basic set of constraints that we will seek to meet in the design of a thick-shelled vacuum vessel. Examining the behavior of the functions $(R-r)^{2} / R^{2}$ and $\left(R^{3}-r^{3}\right) / R^{3}$, which control stability and strength respectively, we can determine that strength will place the stronger constraint upon necessary material behavior. 
Assuming strength limited design, we can combine the mass and strength requirements. Cursory examination of these requirements show that they both scale with $\left(R^{3}-r^{3}\right) / R^{3}$. Isolating this value, we arrive at

$$
\frac{R^{3}-r^{3}}{R^{3}} \leq \frac{\rho_{\text {air }}}{\bar{\rho} \rho_{s}}
$$

for the mass requirement and

$$
\frac{R^{3}-r^{3}}{R^{3}} \geq \frac{3 P_{\text {air }}}{2 \sigma_{s} B \bar{\rho}^{b}}
$$

Combining, we can now write

$$
\frac{3 P_{\text {air }}}{2 \sigma_{s} B \bar{\rho}^{b}} \leq \frac{R^{3}-r^{3}}{R^{3}} \leq \frac{\rho_{\text {air }}}{\bar{\rho} \rho_{s}}
$$

which requires that

$$
\frac{3 P_{\text {air }}}{2 \sigma_{s} B \bar{\rho}^{b}} \leq \frac{\rho_{\text {air }}}{\bar{\rho} \rho_{s}}
$$

Assuming ideal lattice behavior, we can rearrange 26 so that

$$
\frac{P_{\text {air }}}{\rho_{\text {air }}} \leq \frac{2 B \sigma_{s}}{3 \rho_{s}}
$$

represents a clear requirement on the specific strength of the lattice constituent solid material. Evaluating this expression for ideal lattice behavior and atmospheric pressure and air density gives the requirement

$$
1.22 \frac{\mathrm{MPam}^{3}}{\mathrm{~kg}}=1220 \frac{\mathrm{kNm}}{\mathrm{kg}} \leq \frac{\sigma_{s}}{\rho_{s}}
$$

Composites are available today with tensile strengths of $3.82 \mathrm{GPa}$ at densities of $1930 \mathrm{~kg} / \mathrm{m}^{3}$, which would meet the above safety factor.

\section{Thin-Shell Balloon}

The analysis of a thin-shell vacuum balloon is available in the literature [cite], but extended here with cellular material theory. Unlike previous results, we show that even the design of a thin-shell cellular materials vacuum airship is strength limited. As before, the mass of the evacuated balloon must be less than the mass of the volume of the displaced air, which can be written as

$$
\rho_{l}\left(4 \pi R^{2} t\right) \leq \frac{4}{3} \pi R^{3} \rho_{\text {air }}
$$

where $t$ is the thickness of the thin shell. This can be reduced to the relation

$$
\rho_{l} \leq \rho_{\text {air }} \frac{R}{3 t}
$$

Combining the spherical stability requirement with 1 , we write

$$
P_{\text {crit }}=\frac{6 E_{s} A \bar{\rho}^{\alpha} t^{2}}{\sqrt{3(1-\mu)} R^{2}} \geq P_{a t m}
$$

If we assume a perfect lattice $(A=0.1$ and $\alpha=1)$ and combine 30 with 29 , we can write

$$
\frac{R \sqrt{3(1-\mu)} P_{a t m}}{2 t E_{S}} \leq \frac{E_{s}}{\rho_{s}}
$$

For our assumed values and $\mathrm{R} / \mathrm{t}=10$, this reduces to the requirement

$$
\frac{E_{s}}{\rho_{s}}>5.94 \frac{M N m}{k g}
$$


Carbon fiber composites are available with order of magnitude higher relative performance than this requirement. Therefore, we next check the strength requirement. The stress in a thin-shell spherical pressure is given by

$$
\frac{P_{a t m} R}{2 t}<\sigma_{l}
$$

This can be combined with the mass requirement to yield a requirement for the specific strength of the lattice, such that

$$
\frac{3 P_{\text {atm }}}{2 \rho_{\text {air }}}<\frac{\sigma_{l}}{\rho_{l}}
$$

For the assumed values in this analysis, this gives a requirement that the specific strength of the lattice be higher than $124 \mathrm{kNm} / \mathrm{kg}$.

$$
\frac{3 P_{a t m}}{2 B \rho_{\text {air }}}<\frac{\sigma_{s}}{\rho_{s}}
$$

Evaluating using assumed values, we find the requirement

$$
1236 \frac{k N m}{k g}<\frac{\sigma_{s}}{\rho_{s}}
$$

This the same strength requirement obtained for thick-shell design. The result is different from the result found in [14], which note that $E / \rho^{2}$ of the shell material must be greater than order of $7.25 \mathrm{e} 5 \mathrm{Nm} / \mathrm{kg}$. As can be seen in 2 , the scaling of lattice materials results in a requirement on $E / \rho$, which can be met with current materials. A similar plot demonstrates that while shifting to lattice materials changes the scaling of specific modulus requirement, it does not change the characteristic specific strength requirement. Therefore, no matter the relative density,the same margin applies to the lattice strength. Given the available strength of materials when compared to typical moduli, it is clear that lattice vacuum airships will tend to be strength dominated.

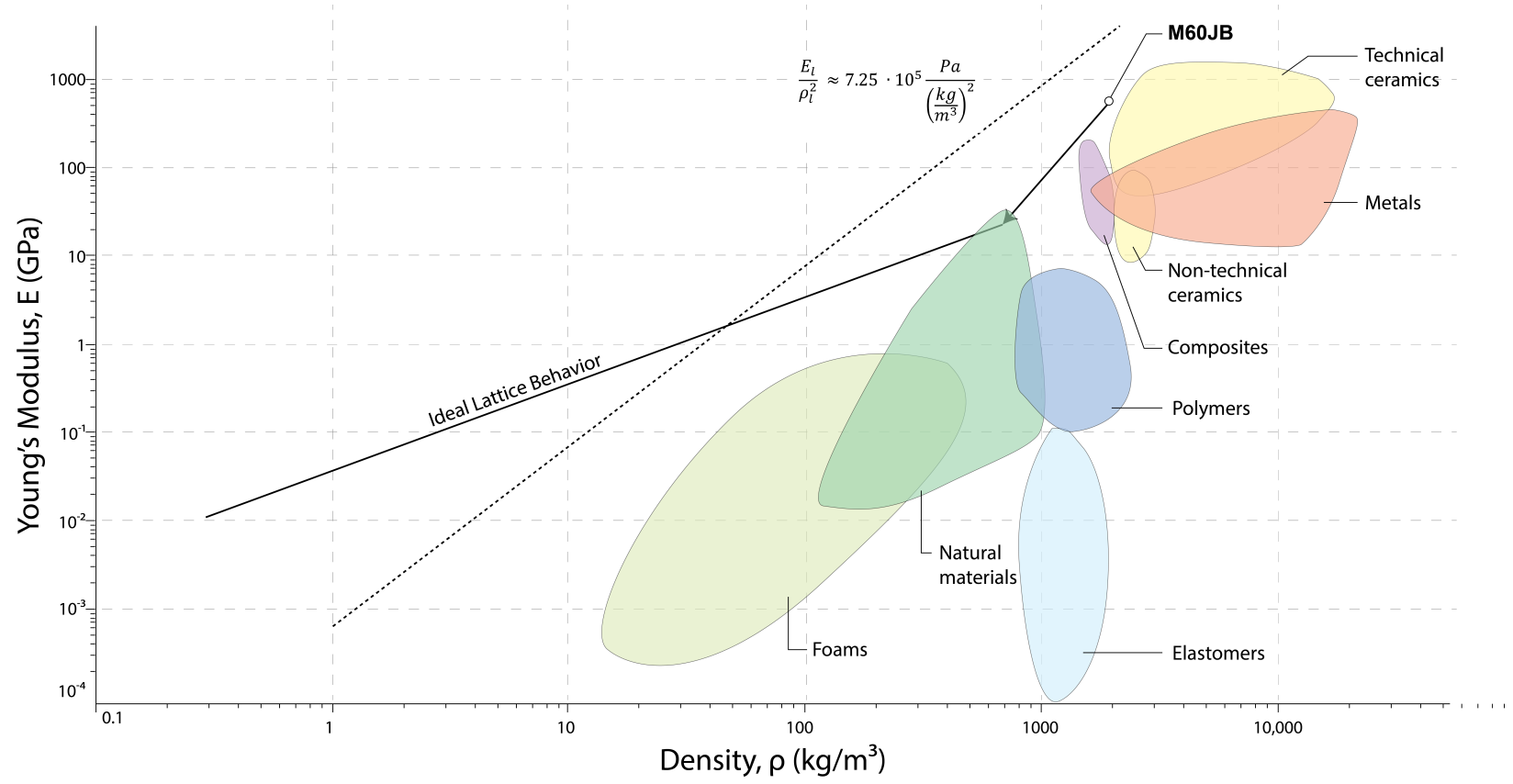

Fig. 2 Young's Modulus vs. density for available materials. The dotted line shows the thin shell stability requirement of the lattice material (as derived by [14] and same order of magnitude as the criterion derived in this work). An example high-performance, commercially available carbon fiber composite (Toray M60JB, tensile strength $3.82 \mathrm{GPa}$, tensile modulus $588 \mathrm{GPa}$, density $1.93 \mathrm{~g} / \mathrm{cc}$ ) is shown on the plot. The arrow and solid line show the performance of an ideal lattice constructed from the example high-performance composite. The arrow shows the performance reduction associated with $A$ in 1 


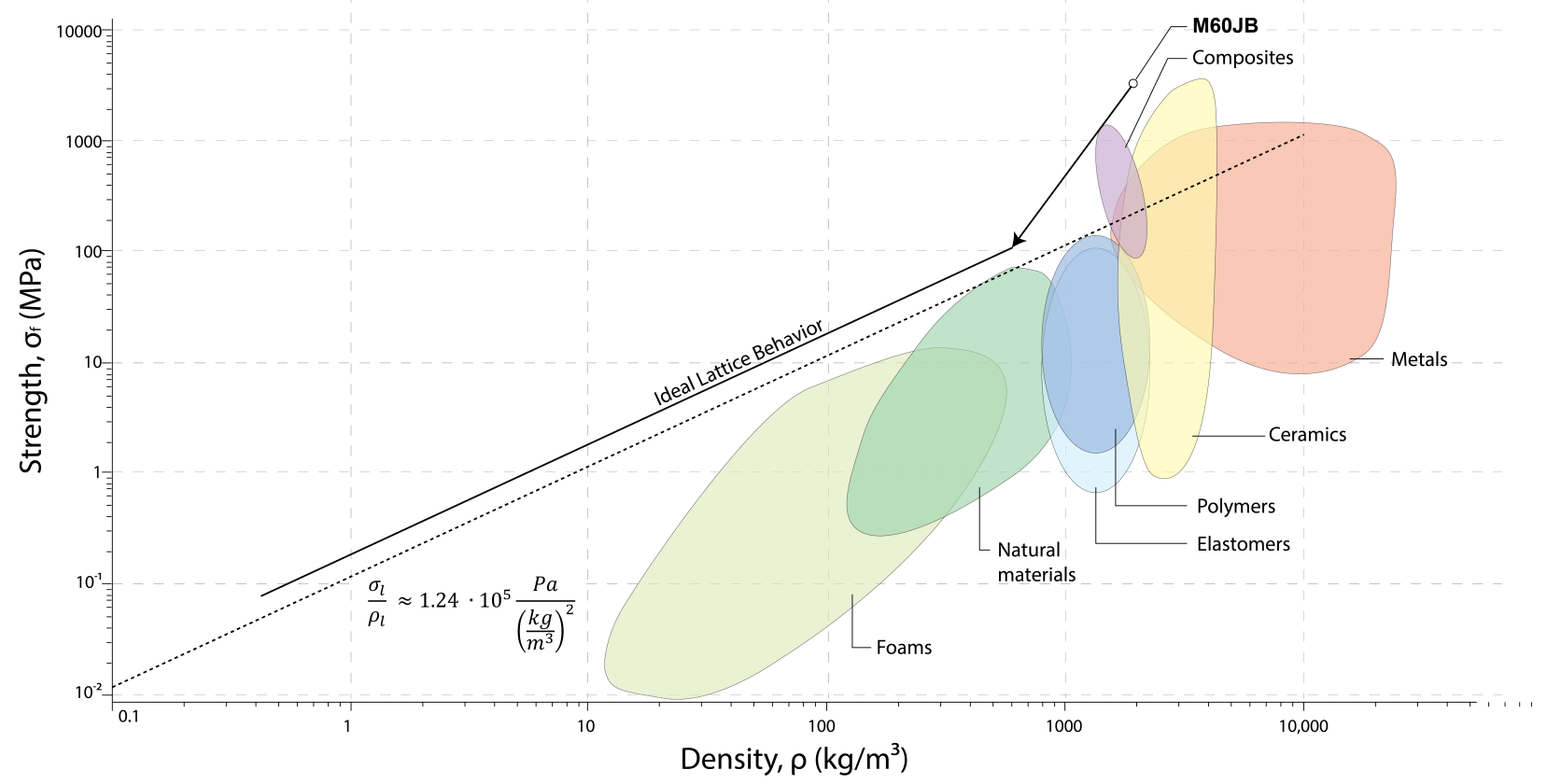

Fig. 3 Strength vs. density for available materials. The dotted line shows the thin shell strength requirement of the lattice material, which is approximately the same as the thick shell requirement. An example highperformance, commercially available carbon fiber composite (Toray M60JB, tensile strength $3.82 \mathrm{GPa}$, tensile modulus $588 \mathrm{GPa}$, density $1.93 \mathrm{~g} / \mathrm{cc}$ ) is shown on the plot. The arrow and solid line show the performance of an ideal lattice constructed from the example high-performance composite. The arrow shows the performance reduction associated with $B$ in 2 .

\section{Design}

Our design methodology is as follows, and is applicable to an airship of any size. First, we impose a 1:10 ratio between shell thickness and radius. Next, we set the lattice pitch to be $1 / 10$ of the thickness. This ensures we achieve continuum behavior. Next, because we can assume there is a uniform stress distribution (which will be shown in the following section), we can use the calculation for shell stress (33) at any given location along the shell, then use this value to drive the design of our lattice. This can be understood as a strength-based design approach, but it is governed by stiffness values of the lattice as we shall show now. The cross sectional stress at a given point is shown to be PR/2t, this can be understood as a force/area, where the area is a square with side length equal to thickness $T$. Because of the 1:10 lattice pitch to thickness ratio, there are 100 lattice elements present to resist this force. Each lattice element receives $1 / 100$ of the force. Because we know the geometry of the lattice element, which in this case is an octahedra, we also know the relationship between an externally applied load along its main axis and the resulting tension and compression forces in its members.

To achieve proper lattice design, we ensure the behavior is governed by strut buckling (as opposed to joint failure). This is acheived by designing the struts based on their expected loads. Having just obtained that value from an initial shell stress, we can now design our struts. Euler buckling is used, and by knowing the force, strut length and Young's Modulus (which for Toray M60JB, E $=588 \mathrm{GPa}$ ), we can then calculate the required second moment of area $I$. This can be used to find the thickness of a square section. By again imposing a 10:1 ratio between radius and wall thickness, we can also design hollow circular tubes, which are much more efficient.

The ability to transition from a continuum material to a discrete beam design is unique to our material system. This approach is shown to be valid by simulating a fully modeled section of the shell, consisting of over 1800 building block parts. 

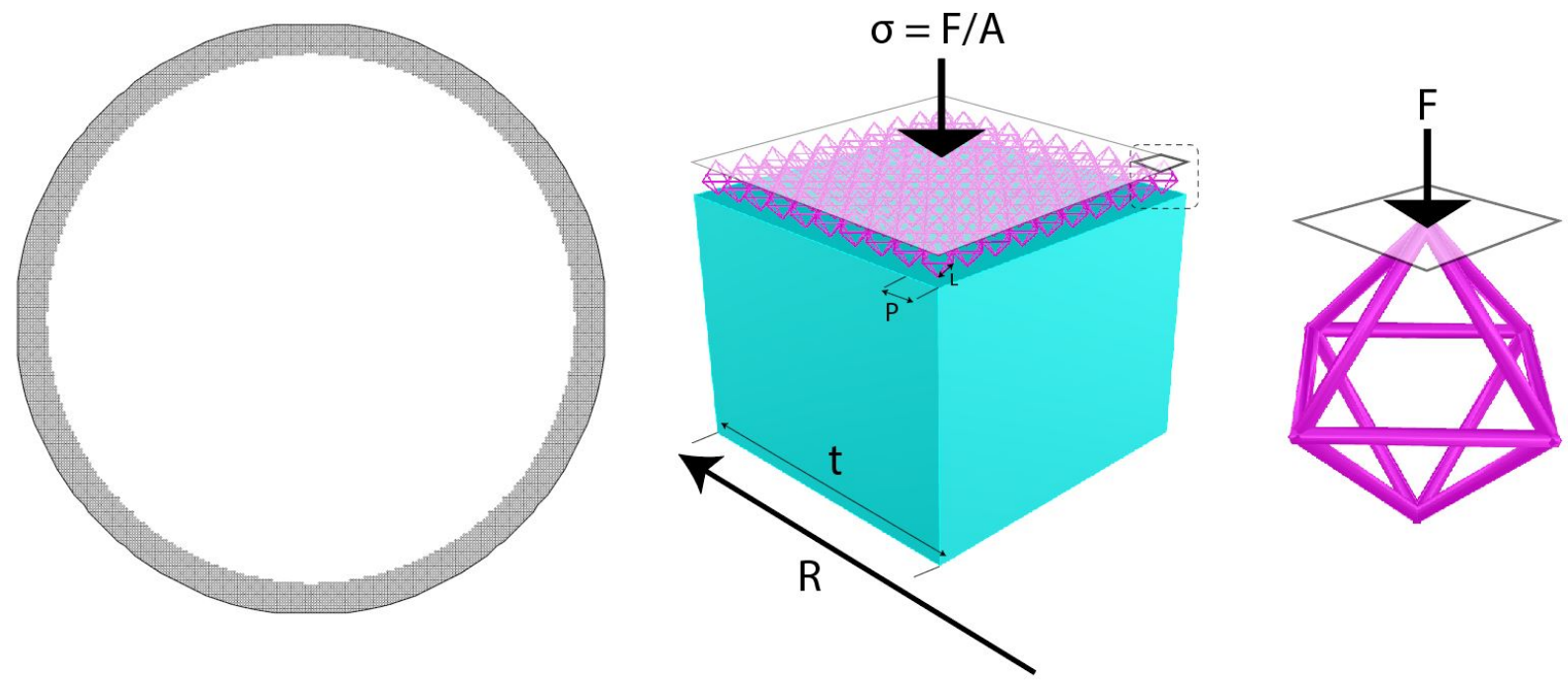

Fig. 4 Design parameters for member sizing. (L to R) 10:1 Radius:thickness, and 10:1 thickness:lattice pitch design rules, and resulting lattice element loading taken from shell stress as a fucntion of force over cross sectional area.
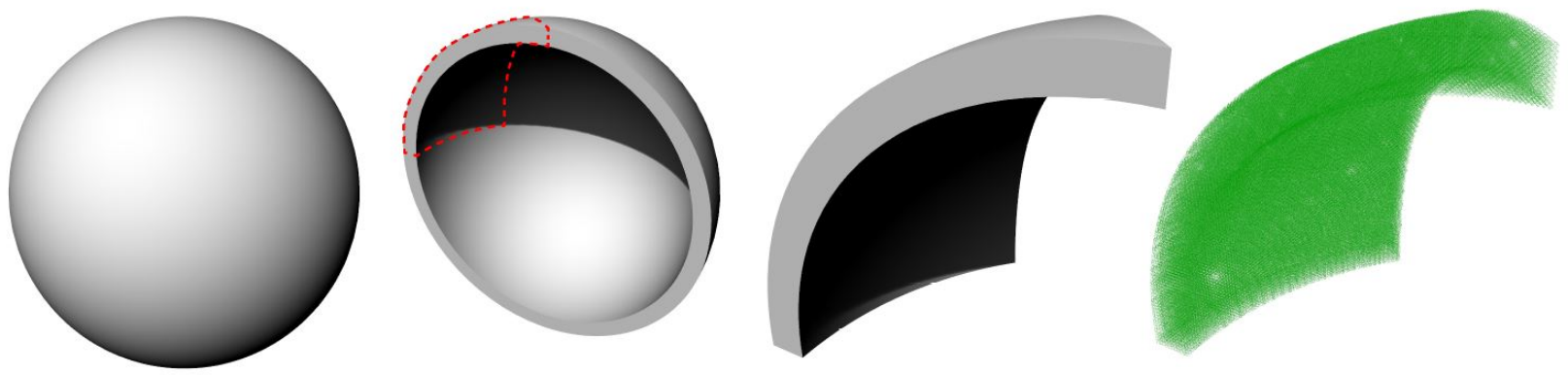

Fig. 5 Views of shell geometry decomposition into lattice materials for part count

\section{Simulation}

In order to test the hypothesis that discrete lattice material will work for a vacuum airship, we wish to numerically investigate its performance. We used the finite element analysis software Oasys GSA, which avoids costly mesh triangulation by performing evaluations of an Euler Bernoulli beam network stiffness matrix. We also limit the simulations here to a planar section of the shell. Full 3D simulations will be performed in later work.

In order to model the boundary conditions of an airship, specifically, that it is floating and thus receives no reactions from any external structure such as a foundation, we need to impose symmetric boundaries across the primary axes of the structure. As shown in 6, we show a structure which is representative of a quarter of a circle. In this case, the nodes are permitted to rotate about the $\mathrm{Z}$ axis (into the page), but are restrained in rotation $\mathrm{X}$ and $\mathrm{Y}$. Similarly, they are restrained from translating in $\mathrm{Z}$, but permitted to translate along the planes of symmetry, which are $\mathrm{X}$ and $\mathrm{Y}$.

As shown in 6, the distribution of axial forces in the lattice beams, which correspond to continuum material stress, are fairly even. This suggests that we should be able to use the lattice material as a continuum material, specifically in this spherical vacuum shell application.

\section{Evaluation}

Using the methods described, we then wish to finally assess the net lift we can generate with a discrete lattice material airship which withstands both global buckling (stiffness) and local failure (strength) criteria. From a given 


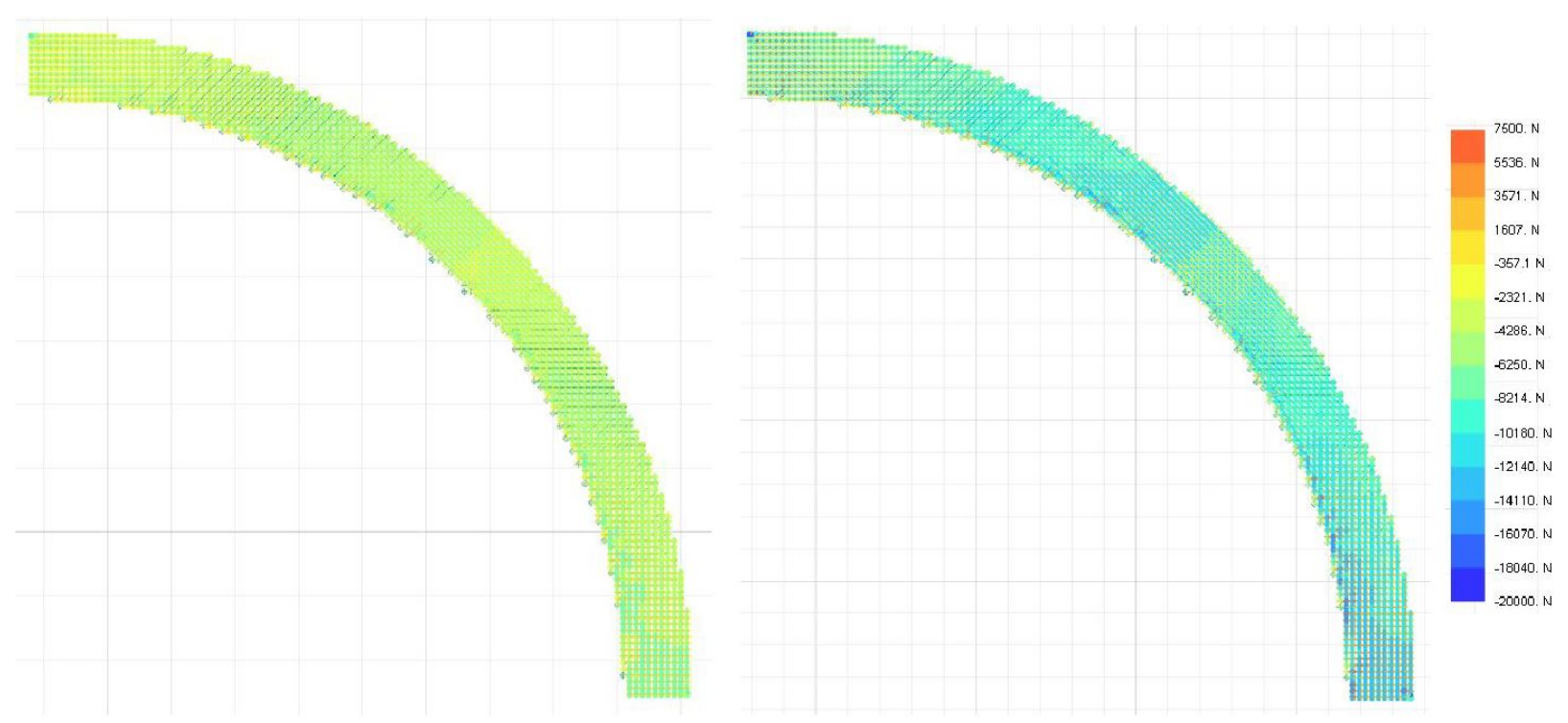

Fig. 6 Simulation results for external pressure loading on a thin shell structure. Axial forces are shown for a $10 \mathrm{~m}$ radius $(\mathrm{R})$ and $20 \mathrm{~m}$ radius $(\mathrm{L})$ sphere

radius, we then calculate the mass of the air displaced when under a vacuum, and based on the structural requirements, we can calculate the mass of the shell. The net lift is the difference between the evacuated air mass and the shell mass. We find that by using hollow tubes, we are able to generate net lift that scales as a function of $R^{3}$

\section{Conclusion}

In this work, we re-evaluate the feasibility of vacuum airships in the context of cellular solids. By applying cellular material scaling laws and taking advantage of developments in architected materials, we show that lattice materials can be used to overcome traditional stability challenges. We concluded that all vacuum airship designs place a stronger requirement on lattice specific strength than lattice specific modulus.

Initial design studies conducted with cuboctahedral discrete lattice materials show that viable designs are possible, with large margins for skin mass penalties.

\section{References}

[1] “Estimated US Energy Consumption in 2017,” https://flowcharts.llnl.gov/content/assets/images/energy/us/Energy $U S_{2} 017 . p n g, ? ? ? ?$

[2] “amazon,”https://patents.google.com/patent/US9305280B1/en, ???? Accessed: 2018-09-30.

[3] "GHG," https://wWw.epa.gov/ghgemissions/sources-greenhouse-gas-emissions ???? Accessed: 2018-09-30.

[4] Nuttall, W. J., Clarke, R. H., and Glowacki, B. A., “Resources: Stop squandering helium,” Nature, Vol. 485, No. 7400, 2012 , p. 573.

[5] Akhmeteli, A. M., and Gavrilin, A. V., "Layered Shell Vacuum Balloons,”, 2006. doi:10.1037/t24245-000.

[6] Fleck, N. a., Deshpande, V. S., and Ashby, M. F., "Micro-architectured materials: past, present and future," Proceedings of the Royal Society A: Mathematical, Physical and Engineering Sciences, Vol. 466, No. 2121, 2010, pp. 2495-2516. doi:10.1098/rspa.2010.0215.

[7] Ashby, M. F., "The Mechanical Properties of Cellular Solids,” Metallurgical Transactions A, Vol. 14A, No. September, 1983, pp. $1755-1769$.

[8] Deshpande, V. S., Ashby, M. F., and Fleck, N. A., "Foam topology: Bending versus stretching dominated architectures," Acta Materialia, Vol. 49, No. 6, 2001, pp. 1035-1040. doi:10.1016/S1359-6454(00)00379-7.

[9] Zheng, X., Lee, H., Weisgraber, T. H., Shusteff, M., Deotte, J., Duoss, E. B., Kuntz, J. D., Biener, M. M., Ge, Q., Jackson, J. A., Kucheyev, S. O., Fang, N. X., and Spadaccini, C. M., "Ultralight, ultrastiff mechanical metamaterials," Science, Vol. 344, No. 6190, 2014, pp. 1373-1377. 


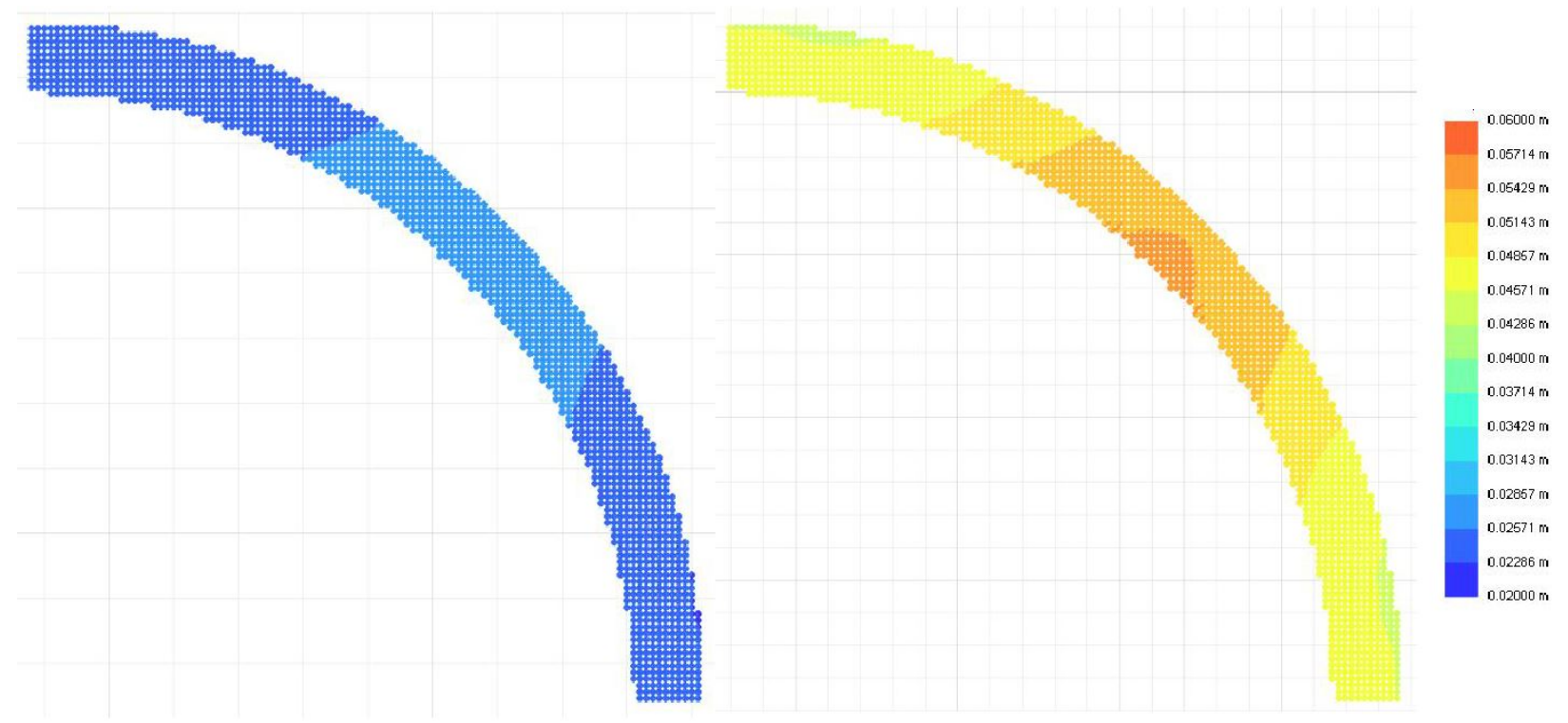

Fig. 7 Simulation results for external pressure loading on a thin shell structure. Displacements are shown for a $10 \mathrm{~m}$ radius $(\mathrm{R})$ and $20 \mathrm{~m}$ radius $(\mathrm{L})$ sphere

[10] Cheung, K. C., and Gershenfeld, N., "Reversibly assembled cellular composite materials," Science, Vol. 341, No. September, 2013, pp. 1219-1221. doi:10.1177/0892705714554493, URL http://wWw.sciencemag.org/cgi/doi/10.1126/science. 1240889

[11] Gregg, C. E., Kim, J. H., and Cheung, K. C., "Ultra-light and scalable composite lattice materials," Advanced Engineering Materials, Vol. 1800213, 2018, pp. 1-6. doi:10.1002/adem.201800213.

[12] Jenett, B., Cellucci, D., Gregg, C., and Cheung, K. C., "Meso-scale digital materials: modular, reconfigurable, lattice-based structures," Proceedings of the 2016 Manufacturing Science and Engineering Conference, 2016.

[13] Berger, J. B., Wadley, H. N., and McMeeking, R. M., "Mechanical metamaterials at the theoretical limit of isotropic elastic stiffness," Nature, Vol. 543, No. 7646, 2017, pp. 533-537. doi:10.1038/nature21075, URL http://dx.doi .org/10.1038/nature21075.

[14] Metlen, T., and Palazotto, A., "Design of a structure that achieves positive buoyancy in air using a vacuum," 54th AIAA/ASME/ASCE/AHS/ASC Structures, Structural Dynamics, and Materials Conference, , No. November, $2013 . \quad$ doi: 10.2514/6.2013-1881.

[15] Latture, R. M., Begley, M. R., and Zok, F. W., "Design and mechanical properties of elastically isotropic trusses," Journal of Materials Research, Vol. 33, No. 3, 2018, pp. 249-263. doi:10.1557/jmr.2018.2. 
Table 1 Discrete lattice airship design parametes

\begin{tabular}{lll}
\hline Parameter & Number of Voxels & Number of Skin panels \\
\hline Value & $1,460,192$ & 125,600
\end{tabular}

Table 2 Spherical Vacuum Airship Lift Scaling

\begin{tabular}{llllllll}
\hline Radius (m) & 0.1 & 1 & 5 & 10 & 20 & 40 & 100 \\
\hline Lift $(\mathrm{kg})$ & 0.003 & 3.0 & 375 & 3005 & 24040 & 192327 & 3005114
\end{tabular}

